\title{
M-Care Child Detection Kiddie Blocks Mobile Application
}

\author{
Lasantha Abeysiri \\ Senior lecturer, Department of \\ Computer Systems Engineering \\ Sri Lanka Institute of Information \\ Technology Colombo, Sri Lanka
}

\author{
R. D. Brahmana \\ Undergraduate, Department of \\ Information Systems Engineering \\ Sri Lanka Institute of Information \\ Technology Colombo, Sri Lanka
}

\author{
W. M. D. M. Wanasundara \\ Undergraduate, Department of \\ Information Systems Engineering \\ Sri Lanka Institute of Information \\ Technology Colombo, Sri Lanka
}

\author{
P. N. Navoda \\ Undergraduate, Department of Information Systems \\ Engineering \\ Sri Lanka Institute of Information Technology \\ Colombo, Sri Lanka
}

\author{
O. V. Godage \\ Undergraduate, Department of Information \\ Technology \\ Sri Lanka Institute of Information Technology \\ Colombo, Sri Lanka
}

\begin{abstract}
This paper is consisting of a theoretical and conceptual framework to improve the child's skill level by implementing a Mobile Application with IT solution which busy parents can get an idea about improving the child's skill level by investigating how child plays with building blocks. Because parents are do not have enough time to pay attention to the child's mental growth and activity skills. And these days there are boring games for children and it is difficult to find a suitable game application for children to play. Just as these days are inactive because they do not play physical games for brain development. Due to these problems, this solution introduces a mobile application that monitors the physical components created by the child and compares the fact physically with the one shown in the application. And decide the current level of the child and customize the game patterns according to the child. Provide evaluation reports and recommend activities to parents that they want to do to improve the child's activity skills in the future. Through this application it is expected to monitors the physical components created by the child and compares that with the one shown in the application and give the feedback. This application will be an integration of 4 main parts and they are implemented to capture the pattern that child built using building blocks, give sample designs, upgrade the game levels, evaluate the child skills, and recommend extra activities.
\end{abstract}

\section{General Terms}

ML - Machine Learning NN - Neural NetworkIP - Image Processing

\section{Keywords}

Skill Level, Building Blocks Designs, Kiddie Blocks, Accuracy Percentage, Performance Level, Recommendations, Machine Learning, Image Processing, Neural Network, Linear Regression.

\section{INTRODUCTION}

Rapid growth of information technology is huge experience at this time. Then the child is the most valuable thing for parents in the world. So parents always trying to give best brain development activates for make them more creative. At current situation brain development process of the children is going on very hard procedure. Most of the physical skill development activates based on the digital equipment. Every human's brain capacity is deciding on during the childhood. Then the gain high capacity and strong mind level and, everyone need good physical skill level development activates covering every side what are need to develop child creativity.

Due the rapid growth of the information technology that emerged, many concepts related to child skill level development and develop child creativity. There is many more skill level development technologies have been introduced both conceptually and practically for those problems. But the accuracy of these systems is still discussed. Most of them are not that practical for some reasons. Main reason is cannot measure the children skill level.

The history of child skill level development systems does not get the expected performance. There are only manual systems and have to capture data. It is too difficult to track the child skill level development process. But in this system real time system capture the child activity and measure the child skill level and give the report for the parents. According to the child's skill level developing system also going with child.

The implementation system will provide the step by step child skill level developing method and monitoring about child's skill level. According to this system child's skill level automatically measure. Then dived stage according skill level of child and help to develop the physical skills of child smoothly.

\section{BACKGROUND}

Nowadays most of existing game apps use same methodologies for increase the Child's skill level, and those apps have not any functions to identify the baby's performance level accurately. All those game apps are basically only giving the activities for the Child.

By the developing M-Care child detection application can overcome those limitations which mentioned above. Therefor this application can help for parent to determine their child skill level and focused on what kind of activities can give them to improve it and on the other hand be alert with their child skill improvement.

When the child $\operatorname{logs}$ in to the application, they need to complete the initial building block designs (default design) first, Then after according to the comparison between the child developed design and initial design, app decide which 
level need to unlock first to play ahead with the game, likewise this game continue up to level twenty. One of the special things in this application is parent can view how their child score at any level of this game and get the recommendation activities advice according to the score. So, in future it hopes to develop this application successfully and give the positive outcome for parent about their child skill level and help to improve it as well.

\subsection{Literature Review}

\subsubsection{Capture the block design and compare it with default design}

In the capture the block design image and compare it with the default design part mainly used the image processing and natural network. In this function uploaded image compare with application level images. [1] Image processing is use to train images which related to the application level images. Comparison done by using the neural network according to the child developed design. Neural network technology decides which level that uploaded image related to the and give the percentage for that.

[2] The important of using neural network is it can handle large amount of data sets and predicate the rate of uploaded image, the usage of neural network is among all trained image data set, that trained model decide uploaded image design level. The important of using Image processing is trained the large amount of image data set which related to each level on the application.

\subsubsection{Identify Child's Skill}

Inside the child skill level identification part mainly using technology is feed forward type neural network. Inside this function, initially give the questionnaire for the mother. The purpose of this is identifying the child's birth condition. Then give the initial three patterns in different difficulty levels for identify the child's current skill level. Inside the child skill level identification part mainly using technology is feed forward type neural network. [4] The expectation using this technology is take the high accuracy output from the trained data model.

The particular child need to complete this initial levels for enter the game. The child's age, complete level of the all three levels percentage takes as the parameters and training the data model. [5] Utilizing the feed forward type neural network trained model can gain high accuracy prediction which level suitable for the child.

There are three levels call easy, medium and hard. System will automatically assign the child to the particular according to child's marks taken for initial level. Then child can start the building designs from that as taking beginning level.

\subsubsection{Performance Evaluation and Progress Report}

Evaluate the child's skills and determine the levels of complexity. Upgrade or degrade the game level and generate the progress report according to the skill level of the child.

In order to level up the child it must analyze the current level, next level to upgrade and previous level to degrade and calculate the number of attempts in the current level. And it must reach a minimum score of $80 \%$. There are so many level-up algorithms to be found with the range and after analysis it decided that Q-learning algorithm is the best for this game. Q-learning is a calculation without supporting learning models. The purpose of Q-learning is to become familiar with an approach that advises a specialist which step to take under which conditions. It does not require a model (hence the implication "without model") of the Earth, and it can handle problems with stochastic changes and rewards, without requiring adjustments. [6]For each finite Markov decision process (FMDP), Q-learning finds a strategy that is ideal because it extends the normal estimate of the full reward to progressive progress, starting with the current status. [7] Qlearning can distinguish an ideal approach to choice of activities for any given FMDP, given unlimited research time and half an irregular policy. [6] "Q" mentions the capacity that benefits the reward that was used to give the fort and it can be said that it represents the "quality" of a movement in a given state. [8]

For the progress recorded graph, the score and the number of attempts are included in one graph and the maximum score and the number of levels that another graph is drawn. Linear regression is used to draw these graphs clearly. By using linear regression, the best fit line is drawn and predicts the score that can be achieved in the next level. Linear regression is a machine learning algorithm based on supervised learning. Perform a regression task. Regression models an objective prediction value based on independent variables. Usually, it is used to track the relationship between variables and predictions. The different regression models differ depending on the type of relationship between the dependent and independent variables they consider and the number of independent variables used. [9]

\subsubsection{Recommendations for the child skill development}

Recommend extra physical activities for the child is most critical part of the paper because every child is different from one child to another from age, skill level and the behavior of the child. To recommend the customized extra physical activities it should analyze data from child's profile. By analyzing child's current skill level, age, and child's health condition it should provide extra physical activities and the system will suggest activities once child reach a minimum score of $65 \%$ of current game level. So to provide accurate and suitable data Feed-Forward Neural Network is the best solution for this function.

A feed-forward neural network is a biologically inspired classification algorithm. It consists of a number of simple neuron-like processing units, organized in layers. Every unit in a layer is connected with all the units in the previous layer. These connections are not all equal; each connection may have a different strength or weight where connections between the units do not form a directed cycle. The weights on these connections encode the knowledge of a network. Often the units in a neural network are also called nodes. This is different from recurrent neural networks. Data enters at the inputs and passes through the network, layer by layer, until it arrives at the outputs. During normal operation, that is when it acts as a classifier, there is no feedback between layers. This is why they are called feed-forward neural networks [10]. 


\section{METHODOLOGY}

\subsection{Capture the block design and compare it with default design}

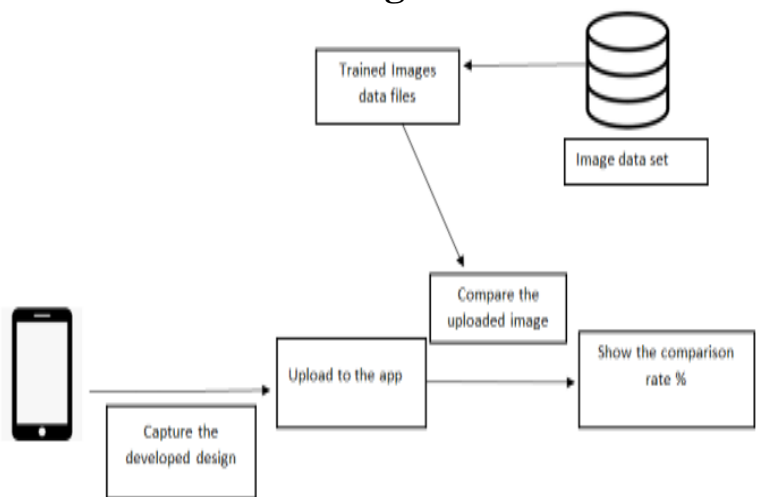

Figure 1: Architecture diagram of image comparison

Capture the image based on the building block design which they need to develop (it's depended on the level that kid fall into) and compare it with selected level image which is include in mobile application.

To capture the design of building block use phone camera and save it in the temporary folder which is help to compare the captured image with the mobile application's level image. All images are in the mobile application up to level 20 trained using image processing and neural network. Therefore, when the user saves the block design, mobile application easily recognizes which level that image can fall into and how closeness that image has.

\subsection{Identify the child current skill level}

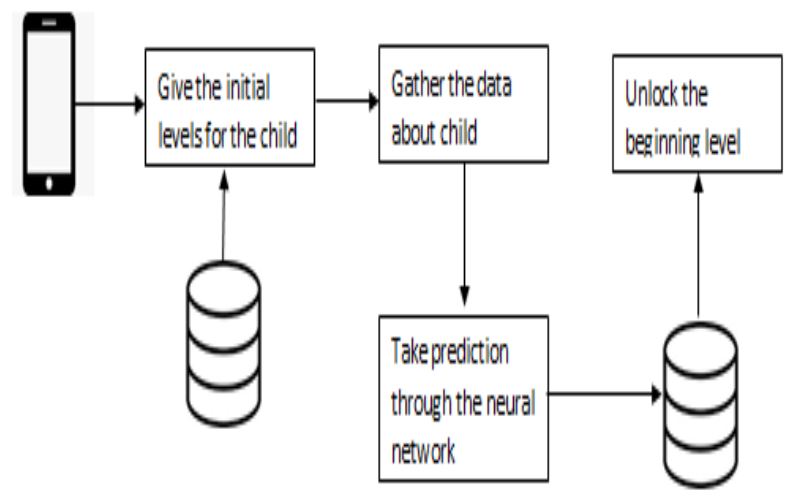

Figure 2: Architecture diagram of skill level recognition

Identify what is the child stay in current level. Initially the system is giving three model levels to child to build and analyze the child's building success rate. The success rate is decided by analyzing the performance of 300 nursery kids. And there are categorized segments under the age, time take for the build a design.

Utilizing this information, process in the neural network and train what are the gained outputs. Then in the mobile application when child buildup and input to system what are the block pattern, analyze the age, time taken to build the block pattern and success rate of the build designs. According to particular output, assign child to what is the suitable stage for start to build their mind level.

Participants - Three hundred randomly selected nursery kids (age range in 3 years to 6 years) were participating for this analysis. They were given pre-designed building blocks designs and asked them to build those designs using building blocks. And capture the time each of them spent to build them and the accuracy of the built designs. Then using the mean of the time and accuracy, calculate the required success rate.

\subsection{Evaluate the progress of the child}

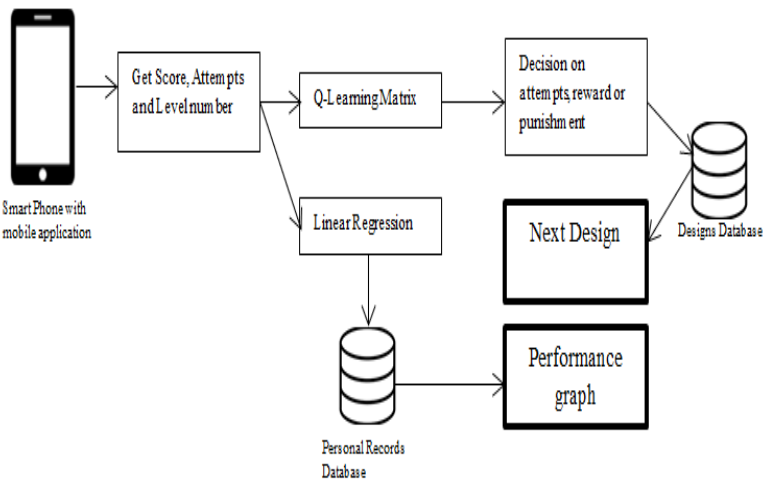

Figure 3: Architecture diagram of level-up and graph

Level up after the child completed expected levels and after obtain the score that match to go for the next level. For this mechanism Q-learning matrix is used and pattern of level upping is no the traditional sequence flow. The Q-matrix is specially design according to this game.

Progress Report-

And each profile of the game adapts to the child. Parents also receive the child's evaluation report and may have an idea on how to improve the child's activity and play skills with basic elements. This part is done by using linear regression and graphs draw in real time according to the child's performance on designs and predict the child's score for next level. And parent can easily recognize whether child is improving or not.

\subsection{Recommendations for the future child brain development}

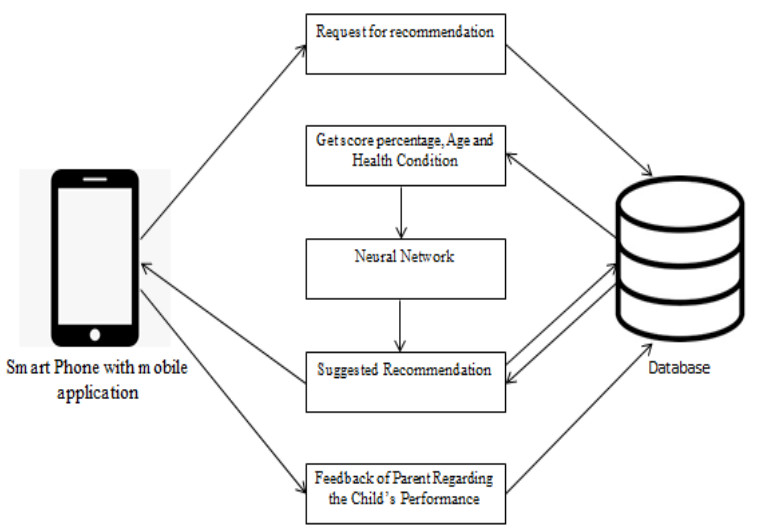

Figure 4: Architecture diagram of recommendations system

The objective of this component is to identifications of the child's current skill level, child will receive physical activities and advices which can help to improve the child skill level. By using neural network and progress graph system will identify the child's current skill level and recommends physical activities and advices which can help to improve the child skill level. Parents can easily click on the recommendations button once child finish with his/her 
particular game level and do the recommended activities with the child. Parents also receive some descriptions with the relevant rate methods to have the clear idea about the activities. All recommendations are depending on the child's age and the percentage of child's skill level.

\section{RESULTS}

\subsection{Capture the block design and compare it with default design model}

Parent need to upload the child developed design to the application to give the comparison between the application image and child developed design image. Uploaded image recognition happens by using the already trained image data model in the application. After the comparison's application determine which level that uploaded image related to and give the comparison percentage according to the child performance in each level of the application.

\subsection{Identify the child current skill level model}

The initially questioner need to fill by parent. Then the child needs the build all the three initial levels are showing by the system. Then using feed forward type neural network, predict which level of child staying. For this process there is trained data model for give the predictions. The predicted value's accuracy rate is always going up to the $90 \%$ range.

\subsection{Evaluate the progress of the child model}

Level-up mechanism is process according to the score achieved for the specific level. Depending on the score, the number of attempts that the decision to upgrade or lower the level. 20 by 20 Q-matrix was trained for this in considering the number of attempts, rewards and punishments. Progress report is drawn using linear regression and a graph is drawn according to the level and the maximum score obtained, while another graph is drawn according to the score and the number of attempts and it predicts the score for the next level with an accuracy of $95 \%$.

\subsection{Recommendations for the future child brain development model}

Provide recommendations are the process that suggests extra physical activities to the child according to the child's performance. Child will receive notifications mentioning suggested activity depending on the child's progress report, age and health condition. Child will allow doing the new activities once he/she will achieve the required performance in relevant level. And parent can update the child's performance with the help of given instructions. The model was able to suggest the extra physical activities with an accuracy of $92 \%$.

Table 1: Accuracy Level of Results

\begin{tabular}{|c|c|}
\hline Name of the Module & Accuracy \\
\hline $\begin{array}{c}\text { Capture the block design and compare it } \\
\text { with default design model }\end{array}$ & $95 \%$ \\
\hline Identify the child current skill level model & $90 \%$ \\
\hline \begin{tabular}{c} 
Evaluate the progress of the child model \\
\hline $\begin{array}{c}\text { Recommendations for the future child } \\
\text { brain development model }\end{array}$
\end{tabular} & $95 \%$ \\
\hline
\end{tabular}

\section{CONCLUSION}

To get to know the child's skill level and get the proper guideline is one of the most important parts when it's come to children's childhood growth. Even the Nowadays lot of activities and technology base devices develop on this title no proper accurate guarantee how far that device can help to improve child skill level while they use that device. To overcome these identified problems, come up with this research project. This mobile application is very accurate and at the same time, it is very practicable, especially this application interface and block design images base on the children's mind guidelines under the consultancy of child skill growth specialist. In this application, the child needs to access the app and finished initial level design images in order to level up on the game. Once the user uploads the child's developed image to the application it will compare the original image which in the application with child developed image, based on the comparison rate application decide which level needs to unlock first to continue the game. One of the important fact in this application is parents can view their child progress Base on the performance of their child at each level. Finally parent can get the proper guidelines base on the performance also. This whole process parent can easily follow-up without having any extra knowledge of technology, that is one of the advantages of using this Kiddie block application and if want kindergarten teachers also can use this application while their free time, to give more meaningful leisure activities

\section{FUTURE WORKS}

It can do lot of additions to the mobile application. This is preparing for android phones and can-do works for bring this to IOS platform. And in this application, it can determine the accurate time that child completed the pattern and finding way for it also needed as well as this game is limited between age 3 to 6 children so hope to expand it for more ages.

\section{REFERENCES}

[1] Besl.P.J and Jain, R.C.1985. "Three-Dimensional Object Recognition." ACM Computing Surveys, Vol.17.

[2] Coin Recognition System using Artificial Neural Network on Static Image Dataset Sakil Ansari1, Dr.V.Kamakshi Prasad2 B.Tech Student1, Professor and Head of Department2 Department of CSE College of Engineering, JNTUH, Kukatpally, Hyderabad, Telangana, India.

[3] Applying the Digital Image Processing to Understand the Role of Compaction in the behavior of ConcreteMixes Ragab M. Abd El-Naby, Emad A. M. El-Dardiry and Naji A. Abo Azzom Civil Engineering Department, Faculty of Engineering at Shoubra, Benha University, Egypt.

[4] $\mathrm{PhD}$ Thesis by Martin Mller Computer Science Department Aarhus University DK8000 Arhus, Denmark November.

[5] Department of Mathematics, College of Education Ibn Al-Haitham, Baghdad University, Iraq Correspondence should be addressed to Ashraf A. T. Hussein; ashraf adnan88@yahoo.com Received 14 May 2013; Accepted 9 June 2013.

[6] J. Abounadi, D. Bertsekas, and V. Borkar. Stochastic approximations for non-expansive maps: Application to Q-Learning algorithms. SIAM J. Control Optim., 41:1$22,2002$. 
[7] Melo, Francisco S."Convergence ” pp. 287-290, IEEE 2010.

[8] Matiisen, Tambet (December 19, 2015). "Demystifying Deep Reinforcement Learning". neuro.cs.ut.ee. Computational Neuroscience Lab. Retrieved 2018-04-06.

[9] Low Y,Gonzalez J, Kyrola A, Bickson D, Guestrin C, Hellerstein JM. Graphlab: A new framework for parallel machine learning. ar Xiv preprint ar Xiv:1006.4990. 2010.
[10] http://www.fon.hum.uva.nl/praat/manual/Feedforward_n eural_networks_1_What_is_a_feedforward_network.

[11] Ilona Bidzan-Bluma and Malgorzata Lipowska. (2018). Physical Activity and Cognitive Functioning of Children: A Systematic Review. Int J Environ Res Public Health. doi: 10.3390/ijerph15040800, PMCID: PMC5923842.

[12] Zan Gao, Senlin Chen, Haichun Sun, Xu Wen and Ping Xiang. (2018). Physical Activity in Children's Health and Cognition. Int $\mathbf{J}$ Environ Res Public Health. doi: 10.1155/2018/8542403, PMCID: PMC6036844 